\title{
EPISTEMOLOGÍA DE LA INNOVACIÓN EN TIC'S DESDE LA VISIÓN DEL MÉTODO DE MORIN
}

\author{
EPISTEMOLOGIA DA INOVAÇÃO DAS TIC A PARTIR DA VISÃO DO \\ MÉTODO MORIN
}

\author{
EPISTEMOLOGY OF ICT INNOVATION FROM THE VISION OF THE \\ MORIN METHOD
}

DOI: $10.22481 /$ rbba.v10i02.8985

Mariangelis del Carmen Gutierrez Nava Universidad Nacional Experimental Politécnica de la Fuerza Armada Nacional Bolivariana Núcleo Falcón Sede Coro, ciudad de Santa Ana de Coro, Estado Falcón, Venezuela ORCID: https://orcid.org/0000-0002-2989-6272 Dirección eletctrónica: mariangelis300191@gmail.com

Ydelbys Rosa Pérez Duno Universidad Nacional Experimental Politécnica de la Fuerza Armada Nacional Bolivariana, Núcleo Falcón Sede Coro, ciudad de Santa Ana de Coro, Estado Falcón, Venezuela ORCID: https://orcid.org/0000-0002-2689-1230 Dirección eletctrónica: ydelbys@gmail.com

\begin{abstract}
RESUMEN
El presente texto propone argumentar el estudio de la gestión de la innovación en TIC desde los principios del pensamiento complejo, así como a la construcción de una propuesta metódica para el estudio de la gestión de la innovación en TIC en empresas públicas basada en la fenomenología moriniana. Se trata de una investigación no experimental de tipo documental en la cual se aplicó, como técnica de investigación, la revisión documental estableciéndose como fuentes de información textos
\end{abstract}


escritos sobre innovación, gestión de la innovación, pensamiento complejo y la fenomenología moriniana. Concluyendo con la identificación una estrecha relación entre la gestión de la innovación en TIC's y la complejidad, lo cual permitió que, dada la epistemología y la ontología del foco de estudio, construir una metodica de investigación basada en la fenomenología moriniana para comprender y explicar este fenómeno.

Palabras clave: Gestión de la Innovación. Tecnologías de Información y Comunicación. Pensamiento Complejo. Fenomenología moriniana.

\title{
RESUMO
}

Este texto se propõe a discutir o estudo da gestão da inovação em TIC a partir dos princípios do pensamento complexo, bem como a construção de uma proposta metódica para o estudo da gestão da inovação em TIC em empresas públicas com base na fenomenologia moriniana. Trata-se de uma pesquisa não experimental do tipo documental em que, como técnica de pesquisa, foi aplicada a revisão documental, estabelecendo como fontes de informação textos escritos sobre inovação, gestão da inovação, pensamento complexo e fenomenologia moriniana. Concluindo com a identificação de uma estreita relação entre a gestão da inovação e da complexidade das TIC, o que permitiu, dada a epistemologia e ontologia do enfoque do estudo, construir um método de investigação baseado na fenomenologia moriniana para compreender e explicar este fenómeno.

Palavras-chave: Gestão de Inovação. Tecnologias de informação e comunicação. Pensamento complexo. Fenomenologia moriniana.

\begin{abstract}
This text proposes to argue the study of ICT innovation management from the principles of complex thinking as well as the construction of a methodical proposal for the study of ICT innovation management in public companies based on Morinian phenomenology. This is a nonexperimental documentary research in which, as a research technique, the documentary review was applied, establishing as sources of information written texts on innovation, innovation management, complex thinking and Morinian phenomenology. Concluding with the
\end{abstract}


identification of a close relationship between the management of ICT innovation and complexity, which allowed, given the epistemology and ontology of the study focus, to build a research method based on Morinian phenomenology to understand and explain this phenomenon.

Keywords: Innovation Management. Information and Communication Technologies. Complex thinking. Morinian phenomenology.

\section{INTRODUCCIÓN}

La complejidad no es algo nuevo o ajeno a las ciencias, siempre ha estado allí presente, por cuanto se debe comprender que la realidad es compleja no desde la concepción de algo confuso, enredado o complicado sino desde la perspectiva de algo difícil de simplificar o definir con una sola palabra. Asimismo, se debe hacer hincapié en la importancia que posee para las organizaciones el desarrollo de la creatividad y la innovación, como dos de las competencias esenciales de las organizaciones dentro de la realidad compleja al permitirles desarrollar aptitudes para el cambio, facilitándoles moverse de manera estratégica ante la globalización y los cambios del mercado, resaltando como las TIC's en sí mismas poseen tiempos de obsolescencia reducidos lo cual dinamiza su mercado (GONZÁLEZ, 2014).

Asimismo, ha de entenderse que la innovación no es un proceso estático sino que en si misma se relacionada directamente con el cambio, el caos y la turbulencia, por ello es esencial desarrollar en las organizaciones la capacidad de adaptarse a la realidad que las rodea, así la innovación es un proceso constante, continúo, dinámico, no lineal por cuanto la linealidad no se adapta a la creatividad y donde se interrelacionan todos los componentes de la organización como un todo abarcando la transdisciplinariedad para la comprensión de las necesidades de cambio o mejora sobre en las TIC's (GONZÁLEZ, 2014).

Como resultado de lo esbozado, resulta importante profundizar en la epistemología de la complejidad como método emergente para el estudio de la gestión de la innovación en TIC, por ello, este estudio se orientó a argumentar el estudio de la gestión de la innovación en TIC desde los principios del pensamiento complejo, así como a la construcción de una propuesta metódica para el estudio de la gestión de la innovación en TIC en empresas públicas basada en

Revista RBBA $\mid$ Revista Binacional Brasil Argentina Vol. 10, num. 2, dez/2021, p. 298-321 
la fenomenología moriniana. Este artículo se estructura en cinco partes luego de la introducción, una primera parte donde se presenta la problemática de la gestión de la innovación en TIC. A continuación, la segunda parte donde se profundizan en aspectos como son innovación, innovación en TIC y gestión de la innovación. Subsiguientemente, se presentan ideas y planteamientos de Morin respecto a la complejidad, el pensamiento complejo, la epistemología compleja y la fenomenología moriniana. A la postre, se presenta la metódica de investigación propuesta. Y para terminar se presentan las consideraciones finales de la revisión realizada.

\section{El problema de la Gestión de la Innovación en TIC's}

Si bien, la gestión de la Innovación ha sido ampliamente estudiada, encontrándose diversos estudios donde destacan la importancia de la Innovación en TIC's; como es el caso de Villalobos (2017), quien afirma que la aplicación de modelos orientados a impulsar la Investigación, Desarrollo e Innovación (I+D+i) en las empresas pueden ser un factor que ayude a desarrollar las capacidades tecnológicas de la empresa, directamente e indirectamente, a través de la mejora de los distintos componentes del capital intelectual, mostrando la estrecha relación existente en Investigación y Desarrollo ( $\mathrm{I}+\mathrm{D})$, innovación y Capital intelectual, así como también la importancia de la I+D+i para impulsar la capacidad tecnológica de las empresas.

Sin embargo, encontramos estudios en referencia al proceso de innovación en América Latina, donde se han detectado un conjunto de debilidades gerenciales. Específicamente en Colombia donde diversos autores realizaron estudios relacionados con la innovación en empresas, tal como Robayo (2015), quien plantea que el proceso de innovación es informal, episódico y no sistemático, orientado a aprovechar las oportunidades de negocio que surgen de las necesidades de los clientes o como estrategias para enfrentar a la competencia. No obstante, a pesar de la existencia de diversas investigaciones que plantean la importancia de la Innovación como proceso sistemático, como por ejemplo los trabajos de Robayo (2015), Villalobos (2017), entre otros, la gestión de la Innovación sobre todo en TIC's continúa siendo un proceso informal, esporádico y no sistemático.

Asimismo, aun cuando existen teorías que involucran la teoría de la complejidad y visión de ecosistema como es la Gestión de la Innovación Total (Total Innovation Management) se ha vislumbrado que esta teoría no ha sido usada como referente en investigaciones en

Revista RBBA $\mid$ Revista Binacional Brasil Argentina Vol. 10, num. 2, dez/2021, p. 298-321 
América Latina, reflexionando sobre el hecho que las condiciones no permiten comparar a realidad de países asiáticos y europeos con países de américa sino que además resulta imposible contrastar la realidad de empresas privadas frente a empresas del estado, fundamentado en que las elementos de la Innovación (factores internos y externos) están sujetas a complejas interacciones e influencias sistémicas, lo que origina que su análisis y comprensión sea un fenómeno complejo, por lo tanto la comparación y generalización resulta difícil (XU et al., 2011; ARGOTHY, 2017).

Por otra parte, se debe resaltar que hasta la fecha no existe un modelo explicativo, generalizable y definitivo que explique el proceso de innovación más aún existen autores que plantean que es cuestionable el tratar de desarrollo un modelo universal del proceso de innovación por cuanto es difícil capturar la complejidad del proceso de innovación en modelos. Aunado a ello, la inexistencia de teorías de gestión de la Innovación con énfasis en el área de las TIC's en empresas del estado venezolano y menos acordes con los principios del pensamiento complejo; se plantea que la comprensión de este fenómeno es una actividad compleja y requiere considerar la influencia del entorno a nivel geográfico, económico, cultural, político, tecnológico, legal e histórico por ello la importancia de una teoría que permita comprender la gestión de la Innovación en TIC's en empresas del estado venezolano (BARRETO y PETIT, 2017; RINCÓN et al., 2014)

Se considera, que algunas de las causas relacionadas con el problema son la visión aislada y desintegradora de la gestión de la Innovación en TIC's, y donde las diferentes áreas de la organización realizan uno u otro proceso de acuerdo criterios particulares sin tomar en cuenta la interrelación con otras áreas de la organización y los posibles efectos de sus acciones sobre los mismos y la organización como un todo. Aunado a ello, la falta de una visión estratégica y una cultura organizacional de innovación que apunte a la gestión de Innovación en TIC's formal y sistémicamente con énfasis en la vigilancia tecnológica. Como resultado, la organización se convierte en un ente pasivo cuyo accionar se orienta a la resolución de problemas, más que a la prestación de servicios innovadores.

Es de resaltar, que a la luz de mi experiencia empírica como investigadora se han podido observar las causas esbozadas anteriormente, pese a esto se plantea que la comprensión de las posibles causas de este problema son más complejas, por una acción de recursividad, por cuanto un efecto siempre se convierte en causa segunda de otros efectos y causa recursiva que modifica la propia causa que lo ha producido, así la falta de motivación en el personal para investigar

Revista RBBA $\mid$ Revista Binacional Brasil Argentina Vol. 10, num. 2, dez/2021, p. 298-321 
producto de la falta de visión estratégica y cultura organizacional de innovación se ha convertido en una causa más del problema que de una u otra manera ha influido en la falta de cultura organizacional de innovación, tampoco se ha considerado hasta los momentos la influencia del entorno en el fenómeno estudiado; por lo que se requiere un estudio más profundo. Justamente porque la innovación no sigue un único camino, es una actividad compleja, diversificada, donde interactúan diversos componentes que influyen en la generación de nuevas ideas, se considera que es posible encontrar un modelo exitoso (ESCORSA VALLS, 2003 como se cita en VEGA et al., 2020).

\section{Gestión de la Innovación en TIC's}

Comenzando con el enfoque económico, la teoría del desenvolvimiento económico concibe como innovación sólo aquel cambio económico espontáneo y discontinúo que se presenta desde el interior de las organizaciones, el cual se cimienta en cambios o desenvolvimientos precedentes quienes se encargaron de crear las condiciones necesarias para este nuevo cambio. En esencia, la innovación se refiere a la puesta en práctica de nuevas combinaciones para la organización, sin importar si esta innovación constituya un descubrimiento científico (nuevo) o la reutilización de un descubrimiento anterior (adaptación). Las nuevas combinaciones pueden abarcar cinco categorías a saber: la introducción de un nuevo bien, desconocido para los consumidores; introducción de un nuevo método de producción, puede ser no probado en la rama económica sobre la cual este trabajando; apertura de un nuevo mercado, donde no haya incursionado; creación de una nueva organización; creación o anulación de monopolio; o, la conquista de una nueva fuente de aprovisionamiento de materia prima o bienes semi-manufacturados (SCHUMPETER, 1957).

Sin embargo, en el enfoque evolucionista, la innovación se reconoce como un proceso contínuo siguiendo las premisas de la teoría Darwinista de la evolución biologicista donde las rutinas en las organizaciones son vistas como los genes en biología. Es decir, características propias de los organismos (organización) que determinan su comportamiento, de manera que las mismas sean heredables y seleccionables tanto por las experiencias favorables como por descubrimiento (NELSON y WINTER, 1982).

De allí que, estas rutinas mejoran la capacidad de las empresas de adaptarse a ciertos cambios en el entorno generando beneficios para las mismas. Por lo cual, la selección de un medio ambiente favorable determina el uso posible de las nuevas tecnologías y la clase de I+D

Revista RBBA $\mid$ Revista Binacional Brasil Argentina Vol. 10, num. 2, dez/2021, p. 298-321 
a emprender por la organización. Asimismo, se destaca cómo la competencia permite a los negocios establecer límites involuntarios pero necesarios para la sobrevivencia (Nelson y Winter, 1982). Asimismo, resulta importante establecer las diferencias entre la innovación tecnológica y la innovación en tecnología. La primera implica el uso de la tecnología como medio para la creación, mejora, desarrollo, uso, difusión de un producto, proceso o servicio, mientras la segunda hace referencia a la generación y puesta a punto de tecnologías nuevas tecnologías que posteriormente serán empleadas para la generación de innovaciones tecnológicas de productos y procesos (Comisión de Normalización y de Fiscalización de Barreras Comerciales No arancelarias - INDECOPI, 2017: 5-6).

En pocas palabras, mientras la innovación tecnológica usa la tecnología existente para mejorar los productos o procesos, la innovación en tecnología se ocupa de la generación o mejoramiento de la tecnología para su posterior uso en la innovación tecnológica. Como consecuencia de lo anterior se tiene que, la innovación en las tecnologías de información, conlleva a la trasformación social por ser capaz de generar la expansión de la calidad/cantidad de información manejada y aumentar la capacidad de almacenamientos. De esta manera, las TIC's transforman la economía, mercados, estructura de las industrias, productos, servicios, puestos de trabajo, y el mercado laboral, ofreciendo a los gerentes una nueva manera de: trabajar, producir, administrar, vender, y brindar sus servicios al consumidor e incluso la necesidad misma de crear productos con tecnología mejor y más competitiva en el mercado (DRUCKER, 1969; OCDE, 2016; VALDERRAMA y NEME, 2011).

Aun cuando no existe de una definición formal sobre Innovación en TIC's, partiendo de la deducción de las TIC's como tecnologías se define la Innovación en TIC' como la actividad de generación y puesta a punto de nuevos o mejorados materiales, dispositivos (hardware), procedimientos, sistemas (software), telecomunicaciones o servicios, destinados a capturar, transmitir y desplegar datos e información electrónica. Es de resaltar que, la innovación constituye un recurso estratégico requiriendo ser gestionado, por ello el objetivo de la gestión de la innovación se fundamenta en suscitar estructuras formales y reglas encaminadas a crear las precondiciones para promover la creatividad con énfasis más en la exploración que en la explotación (DANKBAAR, 2003 como se cita en CASTILLO, 2013; ROBAYO, 2015).

Para Brown (1997), la gestión de la innovación no es solo el cambio tecnológico, incluyendo además el cambio en las personas, cultura, comunicación, organización y procesos de negocio. Por ello, la gestión de la innovación requiere de la gestión del conocimiento,

Revista RBBA $\mid$ Revista Binacional Brasil Argentina Vol. 10, num. 2, dez/2021, p. 298-321 
tecnología, recursos, herramientas, sistemas, liderazgo y cultura, todo un proceso social y cultural. Existen teóricos que consideran que la innovación solo es posible con el uso de la tecnología, tal es el caso de Goffin y Pfeiffer (1999), quienes prefieren el término gestión de la innovación tecnológica como un macro-proceso abarcando gestión estratégica de la innovación, gestión de la creatividad e ideas, gestión el portafolio, gestión de proyectos y gestión de personas.

De igual forma, la Comisión de Normalización y de Fiscalización de Barreras Comerciales No arancelarias - INDECOPI (2017), considera la innovación solo a través la I+D, por ello presentan el sistema de gestión de la I+D+i (Investigación, desarrollo e innovación), donde se involucra la gestión de la estructura organizativa, planificación, responsabilidades, practicas, procedimientos, procesos y recursos de manera que permitan desarrollar, implantar, llevar a efecto, revisar y mantener al día las políticas de I+D+i. Para finalizar, Xu et al. (2011), nos habla de la gestión total de la innovación (Total Innovation Management- TIM) basado en el pensamiento sistémico de gestión de la innovación tradicional adicionando estudios de casos en profundidad de empresas de vanguardia tanto en China como en el extranjero; en él se combinan los conocimientos y la coherencia de la visión de gestión de la innovación tradicional con la vista de gestión de innovación de cartera más relevante.

Resulta importante, resaltar que la gestión total de la innovación se basa principalmente en tres áreas distintas de investigación: teoría de la innovación de la empresa, visión basada en recursos (RBV) de la empresa, y teoría de la complejidad; abarca cinco dimensiones (tipos de innovación): estratégica, tecnológica, en gestión, organizacional, institucional y en marketing. Se sustenta en tres principios, el primero incluye la innovación en todos los elementos tecnológicos y no tecnológicos (estrategia, cultura, organización, institución y mercado); la segunda se relaciona con la innovación de todas las personas involucradas; y la tercera es la innovación en todo momento y en todos los espacios (XU et al., 2011).

En síntesis, se considera que la gestión de la innovación en TIC's debe ser entendida como un sistema donde se integran la gestión estratégica, gestión del conocimiento, gestión del capital intelectual (humano, organizativo, tecnológico, negocio, social y emprendimiento e innovación), gestión de la creatividad e ideas, gestión de proyectos, gestión de la cartelera, gestión del marketing, y gestión de la I+D; la misma debe sustentarse en el estudio del entorno a nivel geográfico, económico, cultural, político, social, tecnológico, legal e histórico y sus implicaciones para la organización, tendiendo como objetivo la generación y puesta a punto de

Revista RBBA $\mid$ Revista Binacional Brasil Argentina Vol. 10, num. 2, dez/2021, p. 298-321 
nuevos o mejorados materiales, dispositivos (hardware), procedimientos, sistemas (software), telecomunicaciones o servicios, destinados a capturar, transmitir y desplegar datos e información electrónica.

\section{Complejidad desde la visión del método de Morin}

La semántica propia de la palabra complejo nos lleva a pensar en turbulencia, desorden, incertidumbre y confusión; Morin reflexiona lo complejo como aquello imposible de resumir, en una palabra, de reducir a una idea, de retrotraerse a una ley. De manera más explícita, la complejidad no elimina la simplicidad, integrando en si los modos simplificadores de pensar. Sin embargo, rechaza la mutilación, la reducción, la unidimensionalidad. Como resultado el conocimiento es multidimensional, depende de condiciones físico-bio-antro-socio-culturohistóricas de producción y de condiciones sistémico-lingüístico-paradigmáticas de organización necesitando de la complementariedad de los pensamientos simbólico/mitológico/mágico y empírico/técnico/racional, estos aun cuando se ha vuelvo antagónicos para las ciencias, interactúan y cohabitan en la cotidianidad (MORIN, 1986; MORIN, 1990).

Dada, la naturaleza multidimensional del conocimiento, este conocimiento no puede ser disociado de la vida ni de las relaciones sociales, depende de procesos infra-cognitivos y ejerce efectos e influencias meta-cognitivos. Partiendo de lo planteado anteriormente, la complejidad nos invita al estudio de los objetos dentro de su contexto, con sus antecedentes, con su devenir, desde una visión multidimensional, donde las nociones aparentemente antagónicas son complementarias entre sí, interactuando orden, desorden y organización. De igual forma, lo complejo no significa lo completo, aun cuando el pensamiento complejo busca la articulación entre diversas disciplinas aspirando al conocimiento multidimensional, reconoce que el conocimiento completo es imposible. En síntesis, el pensamiento complejo se debate entre la aspiración de un saber no parcializado no dividido ni reduccionista, y el reconocimiento de la imposibilidad de tener un conocimiento total (MORIN, 1990).

Pero ¿qué es la complejidad?, debemos entenderla a partir del vocablo complexus es decir tejido en conjunto, así la complejidad es un tejido de constituyentes heterogéneos, asociados e inseparables desde la compresión de lo uno y de lo múltiple, este tejido se forma de eventos, acciones, interacciones, retroacciones, determinaciones y azares que conforman la realidad del fenómeno pero desde la accesión de la complejidad como incertidumbre, desorden,

Revista RBBA $\mid$ Revista Binacional Brasil Argentina Vol. 10, num. 2, dez/2021, p. 298-321 
ambigüedad e inextricable, las ciencias se han dedicado a rechazar tales aspectos quedándose solo con los elementos de orden y certidumbre, mutilando la realidad y conllevando a la inteligencia ciega (MORIN, 1990).

Debemos comprender, que la complejidad es un fenómeno de naturaleza cuantitativa, se fundamenta en un número extremo de interacciones e interferencias entre un gran número de unidades o elementos. Conjuntamente, la complejidad mezcla equilibradamente orden y desorden reconociendo la relación lógica, aunque contradictoria entre orden, desorden y organización; así como la incapacidad de encontrar un orden absoluto. Adicionalmente, nos estimula a la reintegración del hombre entre los otros seres naturales para distinguirlo, pero sin llegar a reducirlo, contraponiéndose tanto a la tendencia de borrar la diferencia llevando el objeto a una unidad simple como a la que oculta la unidad porque solo ve la diferencia. Se trata de ir más allá del reduccionismo y el holismo integrando el pensamiento analítico-reduccionista y el pensamiento global, no se trata de la unidad y del todo, sino de una nueva visión la cual integre ambos puntos de vista (MORIN, 1990).

Por otro lado, la complejidad presenta al sujeto y al objeto mutuamente relacionados, el sujeto reflexivo que piensa en la relación sujeto-objeto de manera que se parte del sujeto reflexivo y se llega a la sociedad, a su historia así el hombre es auto-eco-organizador; es decir el sujeto está contenido en el objeto y el objeto está contenido en el sujeto, influyendo de manera recíproca uno en el otro. Así pues, el sujeto influye en el objeto y este es influenciado tanto por el sujeto como por el ambiente; convirtiendo al sujeto y el objeto en entes inseparables sosteniendo una relación de auto-organizador/ecosistema, donde el sujeto se desarrolla siempre condicionado por un ecosistema, pero el ecosistema existe para un sujeto pensante mostrándose una relación recíproca (MORIN, 1990).

Entonces, el conocimiento es una relación de apertura-clausura entre el conocedor y lo conocido siendo al mismo tiempo abierto/cerrado; supone la separación del conocedor respecto a lo conocido, aunado a la separación interna con nosotros mismos; por lo tanto, conocer es producir una traducción de la realidad del mundo exterior, pero somos coproductores del mundo que conocemos, cooperamos con este mundo y a través de esa cooperación se genera la objetividad del objeto. Reintegrar, al observador en la observación a través de la reflexión autocritica permite nutrir el conocimiento; así llegamos al principio de auto-referencia, auto-crítico y autorreflexivo, en síntesis, el conocimiento solo puede enriquecerse a partir de la reflexividad del sujeto cognoscente (MORIN, 1990).

Revista RBBA $\mid$ Revista Binacional Brasil Argentina Vol. 10, num. 2, dez/2021, p. 298-321 
Por esta razón, la epistemología de la complejidad debe situarse entre la incertidumbre y el dialogo así toda incertidumbre debe confrontarse, corregirse entre sí, inter-dialogar; cada sistema se encuentra dentro de un meta-sistema y así sucesivamente se transciende de un metasistema a otro, pero a la vez imperfecto, cada uno posee brechas y rupturas dando apertura a una búsqueda más profunda del conocimiento, de una teoría para sustituir o revitalizar la anterior. Es por esto, que Morin abandera la complejidad en la concepción del conocimiento como imperfecto y no totalizado, en un camino de búsqueda constante e inacabada (MORIN, 1990).

De esta forma, la epistemología debe ser abierta, (auto) reflexiva (auto) considerando tanto las relaciones eco-sistémicas como meta-sistémicas existiendo conexión laxa, pero indispensablemente, entre apertura sistémica y brecha godeliana, incertidumbre empírica e indecibilidad teórica, apertura física/termodinámica y apertura epistémica/teórica. El sujeto solo puede ser concebido desde su eco-sistema y desde un mundo abierto; es decir dentro de un meta-sistema de allí que sujeto y objeto se integran. Desde la ontología, la realidad se concibe como una red formal de relaciones, compuesta por emergencias, interferencia, fenómenos, múltiples elementos autónomos y a la vez inter-relacionado por juegos sistémicos. Se busca, una ciencia para transcender las disciplinas; esto es la transdisciplinaria reconociendo la incertidumbre, lo incierto y la ambigüedad, siendo capaz de articularse y reflexionar sobre el conocimiento (MORIN, 1990).

En resumen, la complejidad se sustenta en siete principios y tres operadores lógicos. El primero operador, es el dialógico, este nos habla de aspectos contradictorios y antagónicos pero complementarios entre sí, siendo necesario uno para que el otro pueda existir; por ejemplo, orden y desorden son antagónicos, pero ambos necesitan del otro. El segundo, es el principio de recursividad organizacional, este principio contradice la linealidad entre causa y efecto, así los productos y los efectos son, al mismo tiempo, causas y productores de aquello que los produce; es resumen los efectos retro-actúan sobre las causas. En la sociedad se asiste, donde los individuos producen a la sociedad que los produce a ellos mismos; Así productos y productores conforman un ciclo autoconstitutivo, auto-organizador, y autoproductor (MORIN, 1990).

El ultimo principio, es el principio hologramático el cual supera las ideas reduccionistas de ver solo las partes y holista de ver el todo, mostrando que el todo existe en las partes, pero al unísono las partes están en el todo. Este principio, reconoce además como aun cuando las

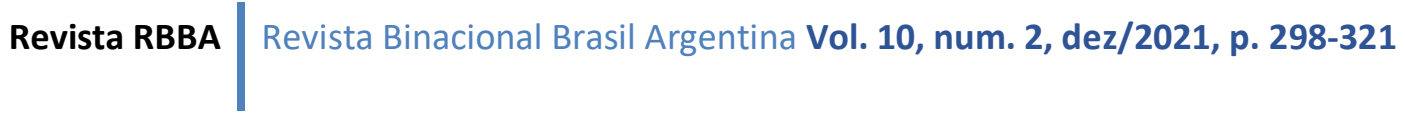


partes están en el todo, existen cualidades emergentes en el todo no presentes en las partes sino procedentes de la interacción entre los diferentes elementos conformantes del sistema, así un todo es más que la suma de sus partes. Asimismo, aun cuando existen conocimientos simples por los cuales podemos conocer las cualidades del conjunto, el todo no es capaz de mostrar las cualidades específicas de todos sus constituyentes siendo al mismo tiempo menos que la suma de sus partes (MORIN, 1990).

En definitiva, la complejidad es el desafío no la respuesta a los problemas, es imperfecta, sabe que la simplificación es necesaria pero esta no debe considerar a la verdad como simple, por lo cual la complejidad se sustenta en la relación entre lo simple y lo complejo, involucrando unir la simplificación presente en la selección, jerarquización, separación y la reducción con los contra-procesos de comunicación, articulación de lo disociado o distinguido superando al pensamiento que solo ve los elementos (reduccionistas) y al que solo ve el todo (globalista). Es el dialogo entre orden, desorden y organización donde las fronteras entre orden y desorden se disuelven, superando las contradicciones sin negarlas, reconociendo la incertidumbre y los problemas, así como la imposibilidad de la totalidad y al mismo tiempo la aspiración a una visión más integral y unificada de la realidad, asociar en ello contradicciones y posiciones antagónicas (MORIN, 1990).

Subsiguientemente, la complejidad es el problema del método del conocimiento para objetos, procesos, comportamiento y sistemas complejos para lo cual es indispensable un pensamiento dialógico, recursivo y holográmatico. Dos procesos importantes para el estudio de los fenómenos complejos, que se convierten en un bucle recursivo son la compresión y la explicación; pero que es comprender, es el conocimiento que aprehende de "aquello" que podemos realizar una representación concreta o una analogía, recociéndose que el conocimiento por comprensión implica subjetividad, afectividad, sentimientos, pensamientos de un ser percibido individuo/sujeto (MORIN, 1986).

Como derivación, comprender es captar los significados existenciales de una situación, acciones vividas, efectuadas, percibidas, de una realidad, de un fenómeno, concebidas por los actores sociales, individuales o colectivos, de manera que las analogías y representación son de naturaleza compresiva. Se entiende la representación como compresiva al permitir formar un análogo del fenómeno percibido pudiendo ser analizada lógicamente. Este conocimiento se ordena mediante representaciones de la realidad o fenómeno, asociando la actividad 
computante y cogitante para producir representaciones, discursos, ideas, mitos, teorías (MORIN, 1986).

Hemos de entender como la representación no es otra cosa que una construcción/transformación/traducción alejada del original, y que la representación perceptiva es al menos, un análogo que aporta la presencia del mundo exterior. El ser humano no percibe la realidad, sino una representación la cual va impregna de lo real y lo imaginario. Aun cuando, la percepción se opone a las visiones imaginarias privilegiando lo real; la representación viene a ser un acto constitutivo idéntico y radical de lo real e imaginario (MORIN, 1986).

Por su parte, las analogías es un conocimiento que surge de la búsqueda de lo semejante por lo semejante que se detecta, de tal manera que se pueden percibir o concebir los objetos o fenómenos en base a sus semejanzas con modelo, esquemas nos permiten conocer. Estas pueden ser por proporciones (similares), relaciones (iguales), formas (isomorfismos), configuración (homeomorifismos), organizacional y funcional (homologías), libres (poesía, lenguaje cotidiano, literatura). Las metáforas por su parte, nos permiten concretar la información mediante el desplazamiento del significado entre dos realidades (MORIN, 1986).

Pero esta compresión no solo emplea las analogías, sino también la proyección/identificación, una proyección de uno hacia los demás y una identificación de los demás en uno (bucle proyección-identificación) esto se debe a que solo podemos comprender lo que sienten los demás al proyectar lo que nosotros sentiríamos en una situación similar. La comprensión emplea, al usar los medios del conocimiento y la comunicación subjetiva se establece una comunicación con el universo subjetivo. El orden y la organización del todo se encuentran en la parte cognoscente, por ello esta puede construir de forma singular y original traducciones ya sea analógicas u homológicas del mundo (MORIN, 1986).

Es importante ver, como la intensidad de estas proyecciones/identificaciones en muchos casos trae consigo la transfiguración de la comprensión en identificación por mimesis, esto trae comprensiones que sin signos aparentes permiten adivinar los sentimientos o pensamientos de los demás de una manera global, esto también conlleva la posibilidad de error y de ilusión al asumir ideas desde la experiencia y no desde la visión objetiva de la realidad, por ende la compresión requiere comprenderse pero sus poderes son insuficientes siendo necesario la explicación de la compresión, por esto se debe combinar la compresión con los procedimientos de verificación y de explicación (MORIN, 1986).

Revista RBBA Revista Binacional Brasil Argentina Vol. 10, num. 2, dez/2021, p. 298-321 
Por lo anteriormente planteado surge la necesidad de la explicación, este es un proceso de abstracto de demostraciones lógicamente efectuadas en virtud de necesidades causales o materiales o formales y/o modelos o estructuras, es decir es situar un objeto o evento relacionándolo con su origen, partes, componentes, constitución, utilidad finalidad. Para poder comprender una representación, esta debe ser organizada de manera coherente de acuerdo a principios/reglas lógicas. Así la explicación depende de la comprensión y viceversa, sin que alguna de ellas domine a la otra (MORIN, 1986).

En este orden de ideas, el conocimiento no es más que traducciones construidas cerebral y espiritualmente, estas traducciones son a partir de señales/signos/símbolos percibidos a través del sistema sensorial. De esta manera nuestra la realidad es nuestra representación de la realidad, y nuestra realidad concebible es nuestra concepción de la realidad, estas no son más que traducciones del bucle sujeto-objeto a la relación espíritu-cerebro. El sujeto es un ser objetivo y objetivable mientras que el objeto de conocimiento siempre estará impregnado de las operaciones, construcciones y traducciones del sujeto. El conocimiento objetivo requiere de la subjetividad presente en el mundo objetivo, así el sujeto está presente en todos los objetos que conoce y la objetividad está presente en el sujeto (MORIN, 1986).

Para finalizar, Morin plantea que la ciencia es consenso y conflictos, se plaga de complejidad donde vemos como el racionalismo y el empirismo se mantiene en conflicto, así como la imaginación y la verificación; la complejidad científica muestra la presencia de lo no científico en lo científico no como mecanismo para anularlo sino para expresarlo, mostrando la imposibilidad de homogenizar. Es indispensables que los investigadores encuentren desde su campo cotidiano un modo de hacer las cosas, de allí que en el discurso moriniano no presentan los eslabones para ir desde sus formulaciones a la práctica, así no existe un único camino definido, sino que debe ser construido por el investigador (MORIN, 1990).

\section{Las redes de relaciones en el método de Morin}

Un paradigma científico puede definirse como el conjunto de principios guía de la investigación en donde se abarcan distinciones-relaciones-oposiciones fundamentales entre algunas nociones matrices que generan y controlan el pensamiento del investigador, así los paradigmas son la constitución de teorías y la producción de los discursos de los miembros de una comunidad científica determinada en un tiempo determinado (MORIN, 1986).

Revista RBBA Revista Binacional Brasil Argentina Vol. 10, num. 2, dez/2021, p. 298-321 
En síntesis, los paradigma indican al investigador el camino que debe seguir, que está haciendo y que se encuentra dentro y fuera de los límites de la investigación, la manera concreta para entender y diferenciar los mismos es en base a tres (3) preguntas, primeramente la pregunta ontológica (¿Cuál es la forma y naturaleza de la realidad y, por lo tanto, que es lo que podemos conocer de ella?), epistemológica (¿Cuál es la naturaleza de la relación entre quien conoce o busca conocer y lo que puede ser conocido?) y metodológica (¿Cómo puede el investigador (el que busca conocer) arreglárselas para averiguar si lo que él o ella cree puede ser conocido?) (GUBA y LINCOLN, 2002 como se cita en BOSCÁN, 2016). De manera que el paradigma se convierte, así, en el principio rector del conocimiento y de la existencia humana (MARTÍNEZ, 2004).

Morin nos muestra una ontología sistémica donde el conocimiento no es más que traducciones construidas cerebral y espiritualmente, estas traducciones son a partir de señales/signos/símbolos percibidos, por ende, la realidad es nuestra representación de la realidad, y nuestra realidad concebible es nuestra concepción de la realidad, estas no son más que traducciones del bucle sujeto-objeto a la relación espíritu-cerebro. En este caso, se considera una ontología sistémica donde la realidad es subjetiva, dinámica, múltiple, hologramática e incierta, La realidad existe la "realidad-en-si" emerge de la interacción entre el sujeto y el objeto esta emerge de la interacción del sujeto y su realidad, y en el cual los elementos no deben ser entendidos de forma aislada, sino a través de las interrelaciones que tienen con otros elementos dentro de una estructura, de manera que la realidad está configurada por sistemas de alto nivel de complejidad interactuando con todas las demás y con "el todo" (MORIN, 1986; MARTÍNEZ, 1997).

Asimismo, epistemológicamente, existe un bucle recursivo y dialógico sujeto-objeto, siendo el sujeto un ser objetivo y objetivable mientras que el objeto de conocimiento siempre estará impregnado de las operaciones, construcciones y traducciones del sujeto de allí que el conocimiento objetivo requiere de la subjetividad presente en el mundo objetivo, así el sujeto está presente en todos los objetos que conoce y la objetividad está presente en el sujeto. En consecuencia, existe una relación subjetiva, el sujeto y objeto se complementan entre sí en la construcción de significado a través de un bucle retroactivo y productor, el sujeto produce al objeto que produce al sujeto asimismo existe una relación transaccional entre el sujeto y objeto, donde los hallazgos son obras creadas a medida que la investigación avanza, el conocimiento se crea solo a través de la interacción entre el investigador y lo investigado (MORIN, 1986).

Revista RBBA $\mid$ Revista Binacional Brasil Argentina Vol. 10, num. 2, dez/2021, p. 298-321 
Por ende, axiológicamente, los valores y emociones están involucrando explícitamente y forman parte de la investigación, reconociendo el papel que juega la cultura, la ideología y los valores del sujeto en la conceptualización y teorización de una realidad compleja estando presentes en la creación de los resultados. De igual forma, metodológicamente se basada en la fenomenología moriniana que camina entre el pensamiento empírico/técnico/racional y el pensamiento simbólico/mitológico/mágico, reconociéndose la importancia de los mitos, las metáforas y los símbolos, para comprender la realidad compleja estableciendo un dialogo entre lo lógico y lo analógico, por ello su interés más por los procesos que por el estado del sistema en un momento dado, siendo así de tipo procesual, empleando instrumentos cualitativos y descriptivos (MORIN, 1986).

Como resultado, en la parte metodológica, la fenomenología moriniana busca comprender y explicar mediante una relación dialógica-sistémica, de allí que se considere que el conocimiento surge de la dialéctica entre el sujeto y el objeto de estudio, siendo no estrictamente “objetivo". Por ello, de acuerdo a lo planteado anteriormente, se encuentra dentro paradigma Emergente, el cual propone un enfoque medular, estructural, dialecto, interdisciplinario y ético, donde cada elemento debe ser estudiado por lo que es y representa, pero además por cómo se relaciona con el resto (MORIN, 1986; MARTÍNEZ, 1997; MARTÍNEZ, 2004).

Por otra parte, el enfoque con el que se observa una realidad depende del punto de vista y a su vez depende del punto de ubicación, así en la investigación este enfoque depende de la opción epistemológica y la opción ontológica. Siendo este de naturaleza dialéctico y sistémico, dialéctico por cuanto el objeto solo puede ser comprendido a través de la interpretación de los sujetos en base a sus experiencias valores, intereses, actitudes y creencias; y sistémico dada la compresión de la realidad como sistema. De manera, que la conceptualización dada de cualitativo en este texto no hace referencia a la naturaleza de las técnicas e instrumentos de recolección de información, sino a la necesidad de identificar la naturaleza profunda de la realidad, su estructura dinámica, aquello que justifica su comportamiento y sus manifestaciones (MARTÍNEZ, 2004)

En este caso particular el proceso de producción del conocimiento es abierto, evolutivo, afronta lo imprevisto y lo nuevo, se encuentran recursos, se puede cambiar el camino en el recorrido por el conocer, planteándose como ruta de investigación un conjunto de fases donde se articulan estrategias, procedimientos y técnicas, que permiten enfrentar, 
describir e interpretar una estructura real dinámica caracterizada por interrelaciones múltiples en múltiples contextos, siendo el criterio de verificación de la investigación, intersubjetivo de naturaleza consensual. En síntesis, hablamos de un método investigación recursivo no lineal, conformado por tres (3) fases de la fenomenología moriniana, como la descripción, comprensión y explicación, empleando como técnicas e instrumentos el análisis de contenido, las entrevistas en profundidad, las analogías, las metáforas, la revisión teórica, el fichaje y la deconstrucción y reconstrucción teórica a partir de la inferencia deductiva y abductiva.

En consecuencia, estas fases forman entre si un bucle dialógico-recursivo-retroactivo no lineal donde una primera fase de descripción que no es más que la transcripción textual de los expresado por los informantes claves. Por su parte, la fase dos (2) denominada comprensiva busca captar los significados existenciales de una situación, acciones vividas, efectuadas, percibidas, de una realidad, de un fenómeno, concebidas por los actores sociales, individuales o colectivos, de manera que las analogías y representación son de naturaleza compresiva Por último, la fase explicativa la cual se basa en entender las causas del fenómeno, el porqué de su comportamiento, así esta fase permite situar un objeto o un evento en relación con su origen o modo de producción, sus partes o componentes constitutivos, su constitución, su finalidad, su utilidad; es situarlo dentro de una causalidad circular y un orden coherente para poder comprender una representación (MORIN, 1986).

Asimismo, resulta indispensable ahondar en el rigor científico de la investigación el cual hace referencia a los criterios utilizados para validar la información, por ello dado el enfoque cualitativo se deben tomar los criterios propuestos por Guba (1989) como son credibilidad, relacionada con la relación entre las realidad construida por los actores sociales y las reconstrucciones verificadas mediante la observación persistente asimismo la saturación a través de la presencia prolongada del investigador en la realidad estudiada. Por otra parte, la transferibilidad dada la naturaleza de los fenómenos y su dependencia del contexto resulta imposible la generalización de los enunciados de la investigación por lo cual los resultados del estudio se delimitarán a su ámbito de estudio en un periodo de tiempo.

Inmediatamente, la dependencia la cual se refiere a la estabilidad de los resultados a través del tiempo, en este caso particular dada el método no lineal, emergente y recursivo de la investigación se considera como una consecuencia, la posibilidad de cambio en las construcciones de los sujetos al largo de la investigación. Para finalizar, la confirmabilidad, referida al proceso de asegurar que los resultados correspondan al contexto de investigación y

Revista RBBA $\mid$ Revista Binacional Brasil Argentina Vol. 10, num. 2, dez/2021, p. 298-321 
este relacionados con los sujetos que hacen vida en el mismo, destacando que los resultados serán las evidencias de la investigación por lo cual es posible garantizar la trazabilidad de los mismos desde las fuentes de información.

Por otra parte, dado el enfoque cualitativo de la investigación, la perspectiva paradigmática y la finalidad de la investigación, así como la naturaleza de los datos que serán recolectados, el análisis de datos de la investigación será un análisis de datos cualitativo. Este tipo de análisis, se orienta a descubrir lo profundo de lo dicho, en lo no dicho, de lo expresado, de lo gestual, es encontrarles sentido a los materiales provenientes de diferentes fuentes, siempre en busca de una compresión e interpretación del fenómeno de estudio. De esta manera, en concordancia con el supuesto teológico y la intencionalidad, la perspectiva teórica metodológica del análisis de datos cualitativo de la investigación se suscribe a la perspectiva interpretativa, misma que pretende captación del significado de un fenómeno o situación, describiéndolo e interpretándolo exhaustivamente (TESCH, 1990).

En consecuencia, para la generación de teoría, se empleará como método de análisis de los datos cualitativos, el análisis de Contenido. Entendiéndose, que el análisis de contenido es una técnica de investigación destinada a formular, a partir de ciertos datos, inferencias reproducibles y válidas que puedan aplicarse a su contexto, conlleva 3 fases: Reducción de datos (reducir el universo potencial de datos al elegir un marco conceptual, preguntas de investigación, los casos e instrumentos), Presentación de datos (ensamble organizado y reducido de información), y elaboración y verificación de conclusiones (determinar significados a partir de los datos presentados) (KIPPENDORFF, 1990 como se cita en TINTO, 2013; MILES y HUBERMAN 1994).

Resulta apremiante declarar, que debido a la gran cantidad de información a generar y la dificultad para su manejo de manera manual, así como en aras de garantizar la credibilidad y confirmabilidad del análisis de los datos cualitativos, se hará uso del software ATLAS.ti. Estos planteamientos constituyen no solo los supuestos epistemológicos de la investigación, sino que además permiten la construcción de la ruta metodológica de la investigación a partir de las fases mencionadas.

Revista RBBA $\mid$ Revista Binacional Brasil Argentina Vol. 10, num. 2, dez/2021, p. 298-321 


\section{CONSIDERACIONES FINALES}

Tal, como lo plantea Morin la complejidad no niega el pensamiento simplificador ni el globalista, sino que nos invita a unir ambas estilos del pensamiento reconociendo como la complejidad no busca obtener una única verdad propiamente (jamás podremos tener un saber total: la totalidad es la no verdad) por el contrario busca es una aproximación a la misma mediante la ampliación y flexibilización del conocimiento, captando la diversidad y pluralidad de visiones donde la linealidad propia de otros métodos simplemente es incapaz es explicar la realidad, de igual forma el método complejo habla de la inexistencia de un camino único sino construido por el investigador a medida que avanza en sus descubrimientos (MORIN, 1990).

De esta forma, podemos comprender como el estudio de la gestión de la innovación en Tecnologías de Información y Comunicación (TIC's) requiere de una visión multidisciplinaria más allá de las ciencias económicas, área donde ha tenido mayor impulso el estudio de la innovación, pero desde una perspectiva más capitalista, mostrándose a la gestión de la innovación como una caja negra, sustentándose su estudio principalmente en entradas (inversión) y salidas (resultados). Por ello, es necesario impulsar el estudio de la gestión de la innovación en TIC's traspasando las fronteras de cualquier área del conocimiento, integrando diferentes perspectivas y visiones.

Resaltando, además como la gestión de la innovación en TIC's al unísono con la complejidad se relaciona con otras teorías como son la gestión estratégica, gestión del conocimiento, gestión del capital intelectual, gestión de la creatividad e ideas, gestión de proyectos, gestión de la cartelera, gestión del marketing, y gestión de la investigación y desarrollo (I+D). De igual forma, aun cuando la innovación no sigue un único camino, es una actividad compleja, diversificada, donde interactúan diversos componentes que influyen en la generación de nuevas ideas, se considera posible encontrar un modelo exitoso (ESCORSA y VALLS, 2003 como se cita en VEGA et al., 2020).

Resulta relevante mencionar, los principios característicos de la complejidad. En primer lugar, el principio holográmatico donde se explica que el todo está en las partes, pero a su vez las partes están en el todo, presentando la idea sistémica y organizacional que aun cuando el todo reproduce cualidades de sus partes, también existente cualidad propios del todo emergentes de las relaciones propiamente. Se vislumbra así la importancia de estudiar la gestión de la Innovación en TIC's, desde pensamiento complejo permitiendo evaluar sistemáticamente

\begin{tabular}{l|l} 
Revista RBBA & Revista Binacional Brasil Argentina Vol. 10, num. 2, dez/2021, p. 298-321
\end{tabular} 
los subprocesos y la trama de relaciones que intervienen en la gestión de la Innovación en TIC's, considerando sistema desde la definición de Morin (1993), como una unidad global constituida de interrelaciones entre constituyentes, elementos, acciones o individuos.

Así, la gestión de la Innovación en TIC's debe ir más allá de los subprocesos aislados apuntalando a la visión de una unidad compleja donde se interrelacionan dichos subprocesos, haciendo énfasis en como la gestión de la Innovación en TIC's no debe reducirse a la suma de sus partes constitutivas, por ende, se requiere comprender como se interrelacionan dichos subprocesos entre sí y con la organización. Igualmente, la gestión de la Innovación en TIC's posee un carácter estratégico no siendo vista como un proceso aislado, separado y particular de cada área de la organización, sino que se integra a otros procesos organizacionales como un todo mediante la gestión estratégica apuntando a la cultura innovadora (ROBAYO, 2015).

Subsiguientemente, el bucle retroactivo visualizando como los efectos se convierten en causas y así se forma un ciclo en el cual los efectos retro-actúan sobre las causas modificándolas. Este principio está íntimamente relacionado con el concepto de auto-ecoorganización, es decir la capacidad del organismo de reorganizarse manteniéndose la unidad aun cuando sus partes se modifican o desaparecen. En este orden de ideas, la gestión de la Innovación en TIC's requiere desde la compresión de las organizaciones como sistemas abiertos adaptables, estas deben vigilar su entorno y recibir información retroactiva de otros departamentos y del ambiente externo, esto es la vigilancia tecnológica necesariamente entendida a través de la causalidad-circular y la retro-alimentación, por cuanto existe influencia del entorno como son los clientes y los diferentes procesos operativos de la organización (MORIN, 1990).

Así, la gestión de la Innovación en TIC's no es un proceso estático requiriendo autoeco-organizarse a partir de la influencia del entorno y la vez, la misma influye en el entorno al generar nuevas expectativas y/o requerimientos en los clientes y/o cambios en la organización, en síntesis el conocimiento y la tecnología resultante de la gestión de la Innovación en TIC's se convierte no solo en capital intelectual para organización sino que terminan siendo la causa de nuevos procesos de Innovación en TIC's. Como consecuencia, el estudio de la gestión de la innovación en TIC's debe sustentarse en el abordaje del entorno a nivel geográfico, económico, cultural, político, social, tecnológico, legal e histórico y sus implicaciones para la organización (MORIN, 1993).

Revista RBBA $\quad$ Revista Binacional Brasil Argentina Vol. 10, num. 2, dez/2021, p. 298-321 
Para finalizar, el principio dialógico que nos habla de la relación y complementariedad de ideas antagónicas y contradictorias como orden y desorden. Dentro de la gestión de la innovación en TIC's, este principio es un elemento clave, por cuanto es indispensable el equilibrio entre las concepciones de cambio y estabilidad. A pesar de afirmarse, como la gestión de la innovación en TIC's debe ser algo constante y permanente, esto no se refiere a la implantación de mejoras incesantemente, lo que conduciría a la imposibilidad de alcanzar la estabilidad, sino al ejecutar actividades de la investigación y desarrollo (I+D) así como a la importancia de la visión estratégica orientada a la cultura de innovación.

Tal como lo explican Van de Ven et., (1999) la innovación no es secuencial y ordenado ni aleatorio; sino que es un ciclo dinámico no lineal de actividades divergentes y convergentes que se repiten en el tiempo y en todos los niveles, siempre y cuando existan condiciones propicias y restrictivas que permitan la continuidad del fenómeno. En ese sentido, el azar es indispensable para poder producir novedad; por tanto, podemos entender a la innovación como el resultado del tetragrama orden/desorden/interacción/organización. Asimismo, dichos autores enfatizan que las personas no pueden controlar el proceso de innovación, pero pueden aprender a maniobrar, esto solo es posible a través de la estrategia.

Entendiéndose, la estrategia a partir de la toma de conciencia del riesgo y la incertidumbre de manera que la organización cambie su rumbo de acción de acuerdo a la información recibida del entorno sacando ventaja de lo imprevisto. Esto se relaciona, directamente con el enfoque evolucionista de la innovación, donde las rutinas en las organizaciones son vistas como los genes en biología por lo cual son heredables y seleccionables tanto por las experiencias favorables como por descubrimiento mejorando la capacidad de las empresas de adaptarse a ciertos cambios en el entorno (MORIN, 1990; NELSON y WINTER, 1982).

En conclusión, la gestión de la innovación es una sinergia entre todos los elementos de la innovación, abarcando la interacción entre la estrategia de innovación y la estrategia de la empresa, así como la integración de todos los recursos para la innovación, hablamos entonces de un ecosistema. De esta forma la gestión de la innovación no es un evento único, sino de alto riesgo, exhibiendo una secuencia interactiva y distribuida no lineal, manifestándose como un sistema extremadamente complejo que requiere coordinación e integración dinámica en tiempo real de estrategia, tecnología, estructura organizacional, procesos de negocio, cultura y 
personas, a sabiendas que los mismos se encuentran en constante cambio y, a menudo, geográficamente dispersos (XU, et., 2011).

\section{REFERENCIAS}

ARGOTHY, L. La innovación en servicios públicos y su contribución al crecimiento endógeno en economías en desarrollo. Un estudio de caso para la economía de Ecuador. 2017. 189 f. Tesis (Doctorado en Economía de la Empresa) - Departamento de Dirección y Economía de la Empresa, UNIVERSIDAD DE León. España.

BARRETO, J., Y PETIT, E. Modelos explicativos del proceso de innovación tecnológica en las organizaciones. Revista Venezolana De Gerencia, Maracaibo, v. 22 n. 79, pág. 387-405, 2017. DOI: 10.37960/revista.v22i79.23028. Consultado en: 29 de junio de 2021.

BOSCÁN, G. Conocimiento, contexto y método. Aspectos que promueven una postura de investigador. Compendium, Barquisimeto v. 19, n. 36, pág. 75-86, 2016. Disponible en: https://www.redalyc.org/pdf/880/88046587005.pdf. Consultado en: 29 de junio de 2021.

BROWN, D. Innovarion Management Tools: A review of selected methodologies, EIMS, 1997. $376 p$.

CASTILLO, Y. Adaptación de un modelo para caracterizar los procesos de gestión de la innovación en las empresas del sector de las TIC de la ciudad de Popayán. Revista Electrónica Gestión de las Personas y Tecnología, Santiago de Chile, v. 16 n. 16, pág. 10-16, 2013. ISSN 0718-5693. https://www.revistas.usach.cl/ojs/index.php/revistagpt/article/view/1137. Consultado en: 29 de junio de 2021.

Comisión de Normalización y de Fiscalización de Barreras Comerciales No arancelarias INDECOPI 2017. NTP 732.001:2017: GESTIÓN DE LA I+D+i. Terminología y definiciones de las actividades de I+D+i, 2. ed. Lima: INDECOPI-CNB, 2017.13p.

DRUCKER, P. The Age of Discontinuity: Guidelines to Our Changing Society. Barcelona: Harper \& Row, 1969. 300p.

GOFFIN, K. y PFEIFFER, R. Innovation Management in UK and German Manufacturing Companies. Anglo-German Foundation, 1999. DOI: 10.1007/978-3-642-18981-4_29.

GONZÁLEZ, I. Las organizaciones trans-complejas: del conocimiento a la toma de decisiones. Revista Venezolana de Análisis de Coyuntura v. XX n. 1, pág. 235-246, enero-junio 2014. Disponible en: https://www.redalyc.org/pdf/364/36433515011.pdf. Consultado en: 29 de junio de 2021.

GUBA, E. Criterios de credibilidad en la Investigación Naturalista. Madrid: Editorial en Gimeno Sacristán Pérez Gómez, 1989. 
MARTÍNEZ M. El paradigma emergente: hacia una nueva teoría de la racionalidad científica, 2. ed. D.F: Trillas, 1997. ISBN 968-24-0415-0. 263p.

MARTÍNEZ, M. Ciencia y arte en la metodología cualitativa. D.F: Trillas, 2004. 351p.

MILES, M. y HUBERMAN, A.M. Data management and analysis methods. En N. Denzin \& Y. Lincoln. (Eds.) Handbook of qualitative research. Londres: Sage Publication, 1994, p. 428444.

MORIN, E. El Método 3 El conocimiento del Conocimiento. Madrid: Catedra Editorial, 1986. 264p.

MORIN, E. Introducción al Pensamiento Complejo. Madrid: Gedisa Editorial, 1990. 176p.

MORIN, E. El Método. Naturaleza de la naturaleza. Madrid: Edic. Cátedra, 1993. 496p.

NELSON, R. y WINTER, S. An Evolutionary Theory of Economic Change. Estados Unidos: The Belknap Press of Harvard University Press. Cambridge, Library Of Congress Cataloging in Publication Data, 1982. DOI: 10.2307/1240942. 454p.

ORGANIZACIÓN PARA LA COOPERACIÓN Y DESARROLLO ECONÓMICOS (OCDE) 2016. Perspectivas de la OCDE en Ciencia, Tecnología e Innovación en América Latina. Paris: OECD Publishing. DOI: 10.1787/sti_in_outlook-2016-1-en. Disponible en: https://www.oecdilibrary.org/docserver/9789264303546-es.pdf?expires $=1625239768 \& i d=i d \&$ accname $=$ guest $\&$ checksum=2D846D9AC80BB26FD8D94F95CFCE8A49. 175p. Consultado en: 29 de junio de 2021.

RINCÓN, E., LEON J., y BARALT, C. 2014. La innovación y el cambio tecnológico desde la perspectiva de la mesoeconomía. ECONÓMICAS CUC, Barranquilla, v. 35, n.2, pág. 89-108. Disponible en: https://repositorio.cuc.edu.co/handle/11323/2725. Consultado en: 29 de junio de 2021.

ROBAYO, P. 2015. La innovación como proceso y su gestión en la organización: una aplicación para el sector gráfico colombiano. Suma de negocios, Bogotá, v. 7, n. 2016, pág. 125-140. DOI: 10.1016/j.sumneg.2016.02.007

SCHUMPETER, J. Teoría del desenvolvimiento económico: Una investigación sobre ganancias, capital, crédito, interés y ciclo económico (Jesús Prados Arrarte trad.), 2. ed. D.F: Fondo de Cultura Económica, 1957 (Obra original publicada en 1937). 256p.

TESCH, R. Qulitative research: Analysisi types and software tools. New York: Falmer Press. 1990. 344p.

TINTO, J. El análisis de contenido como herramienta de utilidad para la realización de una investigación descriptiva. Un ejemplo de aplicación práctica utilizada para conocer las investigaciones realizadas sobre la imagen de marca de España y el efecto de origen. Provincia, Mérida, n. 29, pág. 135-173, 2013. Disponible en: 
http://www.saber.ula.ve/bitstream/handle/123456789/38452/articulo6.pdf?sequence=1\&isAll owed=y. Consultado en: 29 de junio de 2021.

VALDERRAMA, A. Y NEME, O. Efecto de las tecnologías de la información y comunicaciones (TIC) en las exportaciones manufactureras en México. Economía UNAM, México, v. 8, n. 24, pág. 99-122, 2011. Disponible en: http://www.scielo.org.mx/scielo.php?script=sci_abstract\&pid=S1665-

952X2011000300005\&lng=es\&nrm=iso. Consultado en: 29 de junio de 2021.

VAN DE VEN, A., POLLEY, D., GARUD, R. y VENKATARAMAN, S. The innovation journey. New York: Oxford University Press, 1999.

VEGA, V., FERRO, H., RUIZ, M., y BONOMIE, M. Innovación y éxito empresarial: algunas reflexiones teóricas. Revista Venezolana De Gerencia, Maracaibo, v. 2, n. 9, pág. 938-953, 2020. DOI: $10.37960 /$ rvg.v25i91.33175.

VILLALOBOS, L. La Gestión de la Innovación y las Capacidades Tecnológicas: Efectos y Determinantes. 2017. 203 f. Tesis (Doctorado en Ciencias de la Empresa) - Facultad de economía y empresa, departamento de organización de empresas y finanzas, Universidad de Murcia, España.

XU, Q., Jin, L. y Mei, L. Una investigación sobre el mecanismo de construcción de un sistema de gestión de la innovación total a través de la tecnología de la información y la comunicación: estudios de caso en el contexto de China. First International Technology Management Conference, pág. 126-133, 2011. DOI: 10.1109/ITMC.2011.5995937. 